\title{
Growth and development of rats artificially reared on a high or a low plane of nutrition
}

\author{
BY J. L. SMART, D. N. STEPHENS AND H. B. KATZ \\ Department of Child Health, University of Manchester, The Medical School, \\ Oxford Road, Manchester M13 9PT
}

(Received 15 July 1982 - Accepted 20 December 1982)

\begin{abstract}
1. In order to exclude the possibility of differences in maternal care which are known to result from typical methods of undernutrition during the suckling period, rat pups were reared artificially on different planes of nutrition away from their mothers.

2. Artificial rearing was accomplished by fitting infant rats with a gastric cannula through which a milk substitute was infused intermittently. Rats were fed thus from 4 to $21 \mathrm{~d}$ on a high (ARHI) or a low (ARLO; $44 \%$ of ARHI level) plane of nutrition. Underfeeding of the ARLO group was continued till $25 \mathrm{~d}$, after which all rats were given a good-quality pelleted diet ad lib.

3. Compared with mother-reared (MR) litter-mates, ARHI rats showed advanced eye-opening and, at 21 and $25 \mathrm{~d}$, they resisted restraint more strongly.

4. Growth in body-weight of ARHI and MR rats was similar but, when autopsied at 32 weeks, the ARHI rats were shorter (nose-rump length) and had lighter gastrocnemius muscles, adrenals and brains, but heavier epididymal-fat pads.

5. ARLO rats had deficits at 32 weeks compared with ARHI rats in whole body, kidney and epididymal-fat-pad weights, and in tibia length.

6. In a second experiment, ARHI and MR rats were killed at $21 \mathrm{~d}$. All the differences found at 32 weeks were already present at $21 \mathrm{~d}$. In addition, the ARHI pups had enlarged livers and intestines but shorter tibias.

7. The milk substitute, which is one commonly used in such studies, has a low protein and high carbohydrate content compared with rats' milk. This difference probably caused the abnormal organ growth of ARHI rats.
\end{abstract}

Undernutrition of suckling rats results in lasting deficits and distortions of both brain and body growth even after lengthy periods of refeeding (Widdowson \& McCance, 1960; Adlard et al. 1973). Furthermore, adult rates which have been fed ad lib. from weaning after such early-life undernutrition exhibit a variety of behavioural disturbances (Levitsky \& Barnes, 1970; Whatson \& Smart, 1978). It is tempting to relate the altered behaviour of the previously-undernourished rats to the changes observed in their brains, but such an association is at present poorly founded. It is possible that the previously-undernourished rats' abnormal behaviour has non-nutritional causes, namely side-effects of the treatments used to produce nutritional privation (Plaut, 1970). Recently, emphasis has been placed on the finding that female rats behave differently towards growth-retarded pups, thus providing them with rather different early experiences from their well-fed controls (Smart, 1980). Although considerable ingenuity has been displayed in devising a variety of means of rearing undernourished pups, none of the methods has overcome this central problem of altered maternal behaviour towards the malnourished infants. The only technique which appears to offer a satisfactory means of removing this particular confounding factor from the experimental situation is that of rearing infant rats artificially, without their mothers.

The technique introduced by Messer et al. (1969) and modified by Hall (1975) for the artificial rearing of rat pups from the first few postnatal days until weaning, involves fitting the pup with a gastric cannula through which a milk substitute can be infused by some automatic means. Despite the fact that the milk substitute most frequently used for artificial rearing differs from natural rats' milk in several respects, including its lower protein and higher carbohydrate contents (Messer et al. 1969), it has been claimed that artificially-reared 
(AR) rats exhibit normal growth in whole body-weight (Hall, 1975) and in both body-and brain weight (Messer et al. 1969).

We therefore adopted this technique to compare AR rats which received sufficient of the milk substitute during infancy to support normal body-weight gain (ARHI), with rats which were underfed by giving them a restricted amount of the milk substitute (ARLO). Mother-reared rats were also raised to allow assessment of the normality of the ARHI group. In adulthood, after 3 months of ad lib. feeding on a good-quality pelleted diet, various aspects of behaviour were tested which had previously been found to be susceptible to change by early-life undernutrition in normally-reared rats. It was felt necessary to check that the ARHI rats had grown normally, not just in body-weight, but also in terms of the growth of the body's constituent organs. Therefore, rats were killed for autopsy at 32 weeks.

In fact, the growth of several organs was found to be abnormal both in these ARHI rats in adulthood and in others, reared later, at 'weaning'. The latter finding matches and extends the recent report of abnormal organ growth in 18-d-old rat pups artificially reared on the same diet (Diaz et al. 1981). This paper documents, in some detail, the inadequacy of the milk substitute which is currently almost always used for infant rats (Hall, 1975; Ackerman \& Shindledecker, 1978; Diaz \& Schain, 1978; Anderson et al. 1980) in terms of its lasting effects on the growth of several organs. The results of the tests of adult behaviour are not presented, since interpretation would be so highly speculative as to be meaningless in view of the inadequacy of the ARHI animals as a normally-grown control group.

Some of these results have been reported briefly elsewhere (Smart et al. 1981, 1982).

\section{ANIMALS AND METHODS}

\section{Expt 1}

Pups born to Lister hooded rats were left undisturbed in natural litters until $4 \mathrm{~d}$ of age. At that time two male rats were taken at random from each of fifteen litters for artificial rearing, one being fed on a high plane (ARHI) and the other on a low plane (ARLO) of nutrition. A third pup was taken from two of the litters to give a total of sixteen ARHI and sixteen ARLO pups. The remainder of the litter was culled to eight pups, retaining as many males as possible, to constitute a control, mother-reared (MR) group.

The pups taken for artificial rearing were lightly anaesthetized with diethyl ether, fitted with a gastric cannula, according to the method of Hall (1975), and placed singly in expanded polystyrene tubs, $46 \mathrm{~mm}$ deep and $82 \mathrm{~mm}$ base diameter, with wood shavings as bedding. The tubs were floated in a water bath maintained at $42^{\circ}$. When each pup exceeded $13 \mathrm{~g}$ in weight, in order to prevent it from capsizing its tub, the tub was placed into another identical tub which was counter-weighted with a brass bolt through its base. A substitute milk was infused directly into the rat's stomach, via the gastric cannula, from syringes mounted on eight-channel infusion pumps (Model 944; Harvard Instruments, Millis, Massachusetts). The milk (Table 1) was made up substantially as described by Messer et al. (1969), with the major exception being the addition of the bile salt, sodium deoxycholate, which is said to help prevent the gastrointestinal distention known as 'bloat' (Diaz et al. 1980). We found in pilot experiments that intermittent infusion of milk gave better results than the continuous infusion described by Messer et al. (1969) and Hall (1975). Hence, we adopted intermittent infusion schedules: $30 \mathrm{~min}$ infusion in every $2 \mathrm{~h}$ from day 4 to day 16 , and $1 \mathrm{~h}$ in every $3 \mathrm{~h}$ from day 17 to day 21 . ARHI pups received sufficient milk to maintain a gain in weight comparable to that of the MR group, and the ARLO pups received in total $44 \%$ of that amount. The volumes of milk substitute given daily to the ARHI rats rose from $2.5 \mathrm{ml}$ on day 5 , in daily increments of approximately $0.5 \mathrm{ml}$, to $10.5 \mathrm{ml}$ on day 21 . 
Table 1. Ingredients of milk substitute for infant rats

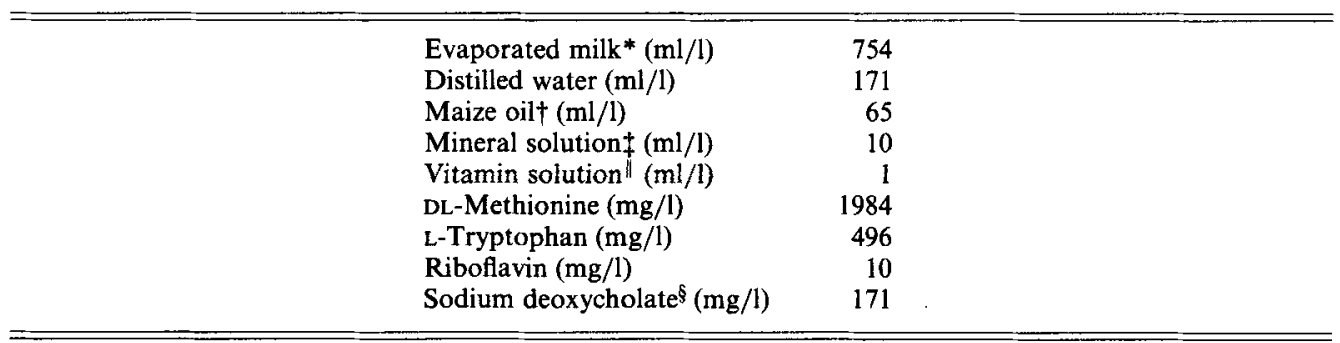

* Ideal Full Cream Evaporated Milk, Nestlé Co. Ltd, St. George's House, Croydon, Surrey.

$\uparrow$ Mazola Pure Corn Oil, CPC (UK) Ltd, Claygate House, Esher, Surrey.

$\ddagger$ Mineral solution $(\mathrm{mg} / 10 \mathrm{ml}): \mathrm{FeSO}_{4} \cdot 7 \mathrm{H}_{2} \mathrm{O}, 27 ; \mathrm{Cu}\left(\mathrm{CH}_{3} \mathrm{COO}\right)_{2}\left(\mathrm{H}_{2} \mathrm{O}\right), 8 ; \mathrm{ZnSO}_{4} \cdot 7 \mathrm{H}_{2} \mathrm{O}, 16$.

* ABIDEC Multivitamin Drops, Parke, Davis \& Co., Pontypool, Gwent $(\mathrm{mg} / \mathrm{ml})$ : retinol 2.00, ergocalciferol 0.017 , thiamin 1.67 , riboflavin 0.67 , pyridoxine 0.83 , nicotinamide $8 \cdot 33$, ascorbic acid $83 \cdot 33$.

$\$$ It has been suggested (Diaz et al. 1980) that addition to this bile salt prevents 'bloating'.

Urination in the neonatal rat occurs only in response to stimulation of the anogenital region. In the absence of the mother this was accomplished twice daily, at about $09 \cdot 30$ and 21.00 hours, by lightly stroking the pups' anogenital area with a small paint brush. Pups were weighed each morning and checked morning and evening for eye-opening on days 12-14 . If any pup was seen to be 'bloated', it was disconnected from the infusion pump until such time as the condition abated. The greatest total loss of milk input incurred in this way for any one pup was $5 \mathrm{ml}$, and this was for an ARHI animal. At $18 \mathrm{~d}$ the pups were transferred to larger tubs ( $51 \mathrm{~mm}$ deep, $93 \mathrm{~mm}$ base diameter) inside counter-weighted tubs of the same size and from this time a wet mash of our normally-pelleted diet (PMD; Labsure Ltd, Poole, Dorset) was made available within the tubs in addition to the infusion of milk substitute.

At $21 \mathrm{~d}$ the gastric cannula was cut close to the body wall and the pups caged in groups of three or four with access, till $25 \mathrm{~d}$, to wet mash and to pelleted diet in unlimited (ARHI) or restricted (ARLO) quantities. Undernutrition ceased at $25 \mathrm{~d}$, from which time all rats were given the pelleted diet ad lib.

MR pups were weighed at $4 \mathrm{~d}$ intervals from day 4 , and were observed for eye-opening each morning and evening on days 12,13 and 14 . They were separated from their mothers at $21 \mathrm{~d}$ anơ caged in litter-mate groups of three or four males. MR rats and their mothers had free access to the PMD pelleted diet throughout.

At 21 and $25 \mathrm{~d}$ pups were rated by two observers for their resistance to handling. Pups were restrained in a prone position on the bench, by holding the head and shoulders with one hand and applying gentle but firm downward pressure on the back with the other hand. Intensity of resistance was scored on a four-point scale: 0 , no resistance; 1 , mild struggling, no vocalization; 2 , struggling and vocalization; 3 , intense struggling and vocalization, may attempt to bite experimenter.

From 17 to 26 weeks rats were subjected to tests of social behaviour (Whatson \& Smart, 1978), operant conditioning (Smart et al. 1973) and maze-running (Katz et al. 1979). They were housed singly from 17 weeks till they were killed at 32 weeks. From 20 to 26 weeks they were given a restricted ration to keep them hungry for the behavioural tests which were dependent on food reward. During this period their body-weight was reduced to $90 \%$ of their free-feeding weight and maintained at that level.

Body and organ measurements. The battery of behavioural tests ended at 26 weeks and animals were again allowed free access to food uritil they were killed at 32 weeks of age. At that time the rats were weighed and their nose-rump length measured under diethyl ether 
Table 2. Stage of eye-opening in 13-d-old mother-reared $(M R)$ rats and artificially-reared rats given either a high $(A R H I)$ or a low $(A R L O)$ plane of nutrition

\begin{tabular}{|c|c|c|c|c|c|}
\hline \multirow[b]{2}{*}{ Expt no. } & \multirow[b]{2}{*}{ Group } & \multicolumn{2}{|c|}{ No. of rats with eyes: } & \multirow[b]{2}{*}{$x^{2}$} & \multirow[b]{2}{*}{$P$} \\
\hline & & Both open & $\begin{array}{l}\text { Not both } \\
\text { open }\end{array}$ & & \\
\hline 1 & $\begin{array}{l}\text { MR } \\
\text { ARHI } \\
\text { ARLO }\end{array}$ & $\begin{array}{l}6 \\
8 \\
2\end{array}$ & $\left.\begin{array}{c}39 \\
4 \\
8\end{array}\right\}$ & $\begin{array}{r}11.808 \\
3.094\end{array}$ & $\begin{array}{l}<0.001 \\
=0.075\end{array}$ \\
\hline 2 & $\begin{array}{l}\text { MR } \\
\text { ARHI }\end{array}$ & $\begin{array}{r}11 \\
7\end{array}$ & $\left.\begin{array}{r}20 \\
3\end{array}\right\}$ & $2 \cdot 390$ & $>0.1$ \\
\hline
\end{tabular}

anaesthesia. They were then exsanguinated and their organs, as listed in Table 3, dissected and measured for weight or length, as appropriate. Selected organs were stored at $-20^{\circ}$. Dry weights of the organs were obtained by drying to constant weight at $110^{\circ}$.

\section{Expt 2}

A second series of ARHI and MR rats was reared as described previously until $21 \mathrm{~d}$ of age to obtain measurements at the end of the period of differential rearing. Sixteen ARHI pups from eight source litters were cannulated on day 4 . The same body and organ measurements were made as in Expt 1 and, in addition, the fat contents of livers were estimated gravimetrically after extraction with chloroform-methanol $(2: 1, \mathrm{v} / \mathrm{v})$.

\section{RESULTS}

\section{Expt 1}

Survival and development. All the MR pups, twelve of the sixteen ARHI and ten of the sixteen ARLO pups survived till adulthood. Two ARLO rats died at $27 \mathrm{~d}$ and it was found on examination that their incisors were abnormally aligned, presumably rendering them incapable of eating pelleted food. Two other rats, one ARHI and one ARLO, showed very poor hair growth by $21 \mathrm{~d}$, but within a few weeks their coats were indistinguishable from the remaining AR rats and from the MR controls.

ARHI pups had both eyes open significantly earlier than MR pups and somewhat earlier than ARLO pups (Table 2). At both 21 and $25 \mathrm{~d}$ the ARHI rats displayed a stronger reaction to handling than MR rats (median scores (range): $21 \mathrm{~d}$, ARHI 2 (2-3), MR $1(0-1)$, $P<0.01 ; 25$ d, ARHI $2.5(1-3)$, MR $1(0-2), P<0.02$, Mann-Whitney U tests). ARLO rats had scores which were intermediate between the other two groups and did not differ significantly from either (21 d, 1 (1-2); $25 \mathrm{~d}, 2(1-3))$.

Body and organ growth. Growth in body-weight of the MR and ARHI rats was similar throughout the experiment (Fig. 1) and differed significantly at only 12 and $21 \mathrm{~d}(t=2 \cdot 111$, $P<0.05 ; t 2.212, P<0.05$, respectively). The ARLO animals weighed less than the ARHI group from day 5 till the end of the experiment (day $5, t 3.233, P<0.01$; day 6 -day 140 , all $t$ values $>4.294, P<0.001$; day 224, $t 2.393, P<0.05$ ), and less than the MR group from day 8 onwards (all $t$ values $>4.198, P<0.001$ ). Their relative weight deficit, which at the end of the period of undernutrition was $41 \%$ compared with ARHI rats, diminished to $9 \%$ at 32 weeks. However, their growth curve diverged from those of the other groups such that their deficit in absolute weight compared with the ARHI group increased from $21 \mathrm{~g}$ at $25 \mathrm{~d}$ to $39 \mathrm{~g}$ at 32 weeks.

At autopsy at 32 weeks the stomachs of all AR rats were found to adhere to the body 

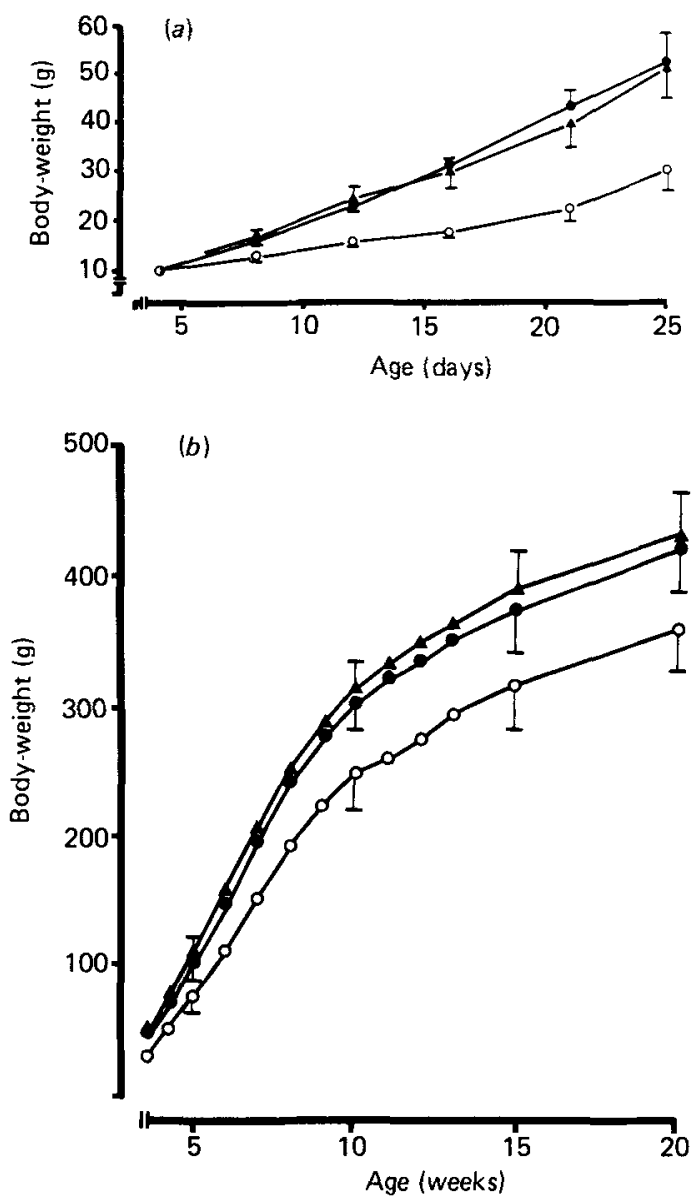

Fig. 1. Body-weights from (a) 4 to $25 \mathrm{~d}$ and $(b) 25$ to $140 \mathrm{~d}$ of mother-reared rats $(\Delta)$ and rats reared artificially from 4 to $21 \mathrm{~d}$ on a high plane $(O)$ or a low plane $(O)$ of nutrition; for details of dietary regimen, see Table 1 and pp. 498-499. Points are mean values and standard deviations are represented by vertical bars.

wall as a result of their earlier cannulation. One of the rats showed atrophy of the stomach and a gross distention of the distal end of the oesophagus, which contained a considerable amount of food, and was further atypical in showing a net loss of weight between 20 and 32 weeks. The results from this rat are excluded.

Table 3 shows the body and organ measurements of the adult AR and MR rats. The previously-undernourished (ARLO) rats, as expected, showed a number of lasting deficits compared with the ARHI animals. They were leaner, as indicated by lower epididymalfat-pad weight, and also had deficits in kidney weight and tibia length, but not in nose-rump length.

Comparison of ARHI and MR rats is more interesting and perplexing. Despite the similarity in body-weights between the two groups, the ARHI rats were significantly shorter in nose-rump length and showed significant deficits in the wet weight of brain, gastrocnemius muscle and adrenal glands; in contrast, their epididymal-fat pads were heavier. There were no differences in liver, kidney and heart weights, nor in stomach weight and intestinal length.

Water content did not differ significantly between groups for any organ. Hence the 
J. L. Smart, D. N. Stephens and H. B. Katz

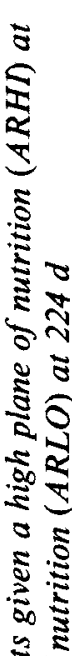

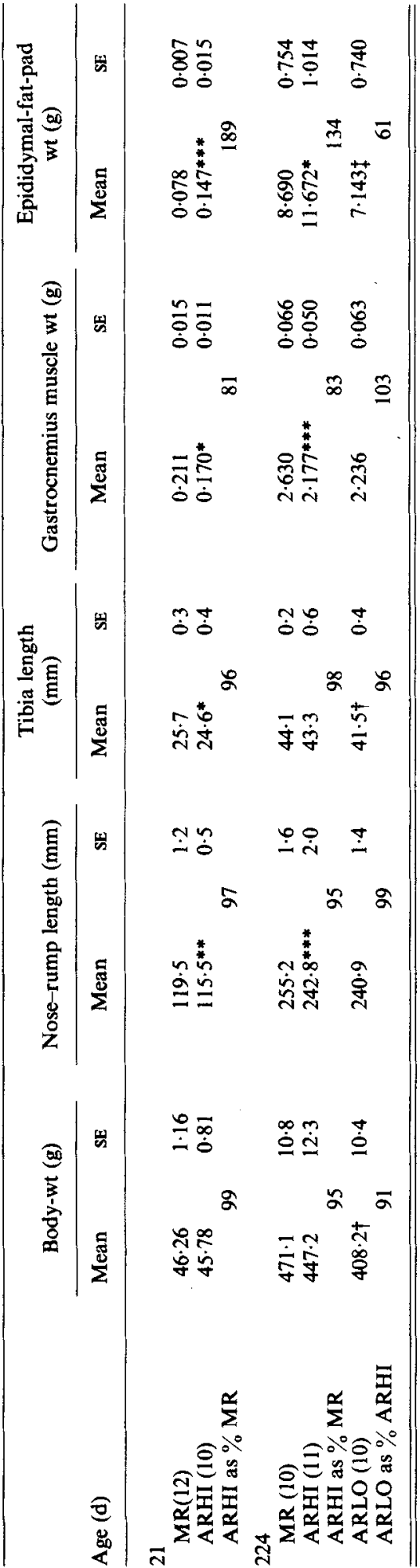

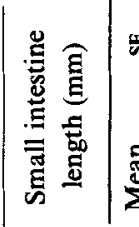

ख

若 营

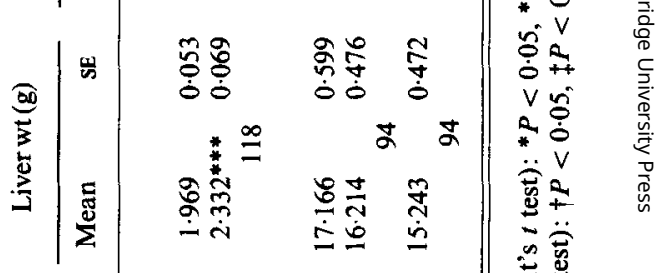

के

ฐ

马

今े है

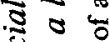

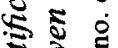

tक

हี

कิ

$\sum$

7

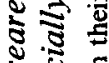

i.

58

ฐ

จ은

3

हैं

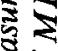

ริ

ป

วิช

ชิ

है

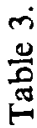


differences in organ wet weights reported previously cannot be explained in terms of inequalities in tissue hydration.

\section{Expt 2}

Survival and development. All MR and ten out of sixteen ARHI pups survived till $21 \mathrm{~d}$. Five of the six non-survivors either died or were killed because of 'bloat'. All surviving pups showed satisfactory hair growth.

Body and organ growth. Eye-opening was somewhat advanced in the ARHI pups, as in Expt 1 (Table 2). All the differences between the adult ARHI and MR rats were already present at $21 \mathrm{~d}$ of age, at the end of the period of artificial rearing. These differences were of similar magnitude at the two ages, with the exception of epididymal-fat-pad weight, in which the excess was relatively much greater in the ARHI rats at $21 \mathrm{~d}$ (Table 3 ). In addition, the 21-d-old ARHI rats had larger livers and greater intestinal lengths, but their tibias were shorter. The differences in weight between the organs from ARHI and MR animals were not attributable to differences in water content, since there were no differences between groups in the water content of any organ. However, the livers of the ARHI pups contained a higher percentage of fat (mean $\pm \mathrm{SE}$ : ARHI $6.95 \pm 0.25$, MR $6.03 \pm 0.19 ; t 2.915$, $P<0.02$ ), though this difference was only a minor component of the difference between groups in liver wet weight.

\section{DISCUSSION}

Despite the fact that the ARHI pups gained body-weight at a similar rate to MR controls, both during the period of artificial rearing and subsequently, the present experiments indicate that the artificial-rearing procèdure, as it exists at present, does not provide a means of rearing rat pups which are comparable in body conformation and organ development to MR animals. In particular, the reduced growth of the brain, as well as the poor growth of lean tissues, and the indications of obesity preclude the use of the technique as it stands to study early nutritional influences on growth and behaviour.

Although it is not possible from the present experiments to identify the reasons for the distorted growth of the ARHI rats, we may point to several features of the diet and of the procedure which may have contributed. The possibility of differences in the volume of milk taken by MR and ARHI rats cannot be ruled out. Unfortunately, no informed comparison can be made since the scientific literature on the milk intake of rats is singularly unhelpful. Most of the evidence depends on the unphysiological procedure of separating pups from their mother for some hours, reuniting them and then test-weighing the pups after they have fed. Estimates of daily intake derived from this procedure cannot be reliable. Recently, two attempts to estimate normal milk intake have used tritiated water to follow water exchange between the dam and her pups, either by measuring the accumulation of ${ }^{3} \mathrm{H}_{2} \mathrm{O}$ in the body water of the pups following administration of labelled water to the mother (Godbole et al. 1981) or by injecting the tracer into the pups and measuring its turnover as it is replaced by unlabelled water from milk. Although, in principle, both techniques appear sound, they give rise to quite different values for milk intake. For instance, daily intake per pup at $10 \mathrm{~d}$ of age was found to be $2.0 \mathrm{ml}$ (Godbole et al. 1981) and $6.7 \mathrm{ml}$ (Coward et al. 1982). Clearly there are differences between these investigations, of a theoretical or methodological nature, which need to be resolved.

Uncertainty, therefore, remains about the appropriateness of the volumes of milk substitute infused, but there can be little doubt that its composition differs considerably from that of rats' milk. Most obviously, the protein content is low compared with rats' milk $(66 v .92 \mathrm{~g} / 1)$ and the carbohydrate content high $(95 v .30 \mathrm{~g} / \mathrm{l})$ (values for milk substitute are calculated from information provided by Nestlé Co. Ltd (Croydon, Surrey) for 
evaporated milk; values for rats' milk from Messer et al. 1969). In our experiment the low-protein content of the diet may have resulted in low total protein intake, thus preventing normal growth of lean tissue and brain. The suckling rat uses its protein intake mainly for growth, with gluconeogenesis accounting for only a small fraction of total protein utilization (Hahn, 1979). Thus even a small reduction in protein intake at this age is likely to lead to growth retardation.

Why the ARHI rats became fat is less easy to explain. Two factors come to mind. First, the energy density of the milk substitute is probably higher than that of rats' milk (7549 v. $6484 \mathrm{~kJ} / \mathrm{l}$ ) (values calculated respectively from information provided by Nestlé Co. Ltd and from Messer et al. 1969 , using the following conversion factors: $1 \mathrm{~g}$ protein $=17 \mathrm{~kJ}$, $1 \mathrm{~g}$ fat $=37 \mathrm{~kJ}, 1 \mathrm{~g}$ carbohydrate $=16 \mathrm{~kJ}$ ), and the disparity may increase with the age of the pups since the fat content of rats' milk is said to fall markedly in the course of lactation (Godbole et al. 1981). The other factor is the abnormal composition of the dietary fat, with a much smaller proportion of medium chain triglycerides than rats' milk which, together with the diet's high carbohydrate content, may be the cause of the abnormal intermediary metabolism of rat pups reared on this diet (Sonnenberg et al. 1982). Such pups show lower than normal levels of ketone bodies in blood, slower turnover of ketone bodies and high insulin and galactose concentrations in blood; there is a greater contribution of glucose and a smaller contribution of ketones than normal as the substance for respiration. Thus, there appears to be a switch from fat to carbohydrate as the major energy source, leaving dietary fat available for storage.

Less obvious dietary factors may also have contributed to the abnormal growth of the ARHI rats. These include differences in the digestibility of the diets, the balance of amino acids, the content of vitamins and minerals and the pattern of meals. However, it is impossible without extensive research to ascertain the appropriate explanation. Indeed, possible explanations need not be limited to nutrition, because differences in the environments of MR and AR rats, such as the very confined living space and unvarying ambient temperature of the latter, might also have some influence on growth.

It is a notable finding that the ARHI rats show abnormalities in the growth of certain organs which persist well beyond the period of artificial rearing; that is, they are present in weanling and in adult animals. This, of course, is not without precedent, since it has long been known that rats undernourished till weaning continue on refeeding to show deficits in organ growth compared with well-fed controls of the same age (Widdowson \& McCance, 1960). What is remarkable about the present findings is that the abnormalities include both deficient organ growth and, in the case of the epididymal-fat pads, excessive growth. Possible causes of the immediate effects of the artificial diet and procedure have already been discussed, but these do not explain why the effects should persist. Such lasting effects have been the subject of much theory and speculation (McCance, 1976; Dobbing, 1981) and the issue remains controversial. Suffice it to say here, that the present results indicate a mechanism which is organ-specific rather than one involving an overall control of growth.

In addition to their altered body composition, the ARHI pups also displayed functional changes. They showed advanced eye-opening compared with their MR brothers and, around the time of weaning, showed greater resistance to restraint. It is less likely that these changes are attributable to the abnormal diet, or at least solely attributable, than to differences in the environments experienced by the AR and MR pups. Various aspects of development have been found to be accelerated by stimulating infant rats and mice in a variety of ways, including handling, gentling, exposure to cold and electric shock (reviewed by Levine \& Mullins, 1968; Daly, 1973). In some studies the additional stimulation has resulted in advanced eye-opening (Levine, 1959; Barnett \& Burn, 1967) and it may be that the much more frequent handling of the ARHI pups in the present investigation was the cause of 
their earlier eye-opening. The rather later eye-opening of the ARLO pups was an expected consequence of their underfeeding, in line with several previous reports (e.g. Smart \& Dobbing, 1971). Several possible mechanisms have been proposed for the 'early stimulation' effect, including mediation through tactile and olfactory stimulation, hypothermia, stress and, most recently, altered mother-infant interaction (reviewed by Russell, 1971). It is important for these considerations to note that the present effect was obtained without maternal mediation and, hence, it is no longer tenable that the mother is a necessary mediating agent.

It is somewhat puzzling that the ARHI rats should show greater resistance to restraint than their MR brothers, in view of the fact that they were handled much more often. Before hypothesizing some neurological or behavioural mechanism, the possibility should perhaps be considered that they may simply have suffered more discomfort during restraint because of the presence of their cannulas.

We have dwelt on the comparison of ARHI with MR rats because of the implications of those findings for other users and would-be users of the milk substitute formulated by Messer et al. (1969). However, the comparison between the two AR groups, ARHI and ARLO, is also noteworthy in at least one respect. In spite of receiving less than half the amount of milk given to the ARHI pups during artificial rearing, the ARLO rats in adulthood differed relatively little from the ARHI group in body and organ growth. They showed deficits only in whole body-weight, tibia length, epididymal-fat-pad weight and kidney weight. Assuming that the $39 \%$ deficit in epididymal fat is an indication of a similarly substantial deficit in whole-body fat, it seems likely that much of the $9 \%$ difference in bodyweight between ARLO and ARHI rats can be attributed to fat. Such other measurements as nose-rump length and the weights of the brain and gastrocnemius muscle were not significantly lower in the ARLO group. Since no ARLO pups were autopsied at 'weaning', there is no information on whether their organ growth was retarded early in life but 'caught up' later with ad lib. feeding, or whether, in fact, they were no more growth-retarded at weaning than were ARHI rats with respect to certain organs. Feeding reduced quantities of the imbalanced milk diet may have had an effect on the growth of most organs which was little worse than feeding the full amount.

A few of the AR rats had specific abnormalities early in development or later. The adult rat which was found at post mortem to have hypertrophy of the distal end of the oesophagus, had presumably suffered some damage to the sphincter during cannulation, resulting in a latent weakness. This became symptomatic only after the period of restricted feeding associated with some of the behavioural testing, when this rat, unlike the others, lost weight. The tendency to take an abnormally large meal on presentation of the restricted daily ration may have further damaged the already-weakened sphincter, causing accumulation of food in the oesophagus perhaps through reflux from the stomach.

The two animals which displayed poor hair growth during artificial rearing both recovered within a few weeks of 'weaning' to become indistinguishable from the other rats, suggesting some sort of dietary deficiency. However, this interpretation is complicated by the fact that one of these was on the high and the other on the low plane of nutrition. Why the ARHI rat should show symptoms whereas all but one of the ARLO pups did not is perplexing. One is left with the unsatisfactory suggestion that individual variability in nutrient requirements is great.

Two other ARLO pups died after transfer to pelleted food and were judged to have been unable to bite hard food because of the abnormal alignment of their incisors. In this case it seems plausible to suggest that this may have been due to lack of use of the teeth during their growth.

The artificial-rearing technique is potentially extremely useful for a variety of develop- 
mental studies, nutritional, pharmacological and psychological. However, the report of atypical intermediary metabolism in AR rat pups (Sonnenberg et al. 1982) and the findings of abnormal somatic development both early (Diaz et al. 1981) and later in life (present study) give rise to serious reservations about its use. The composition of the milk substitute appears to be the likely source of the difficulties, though other environmental factors associated with the artificial-rearing method cannot be ruled out. It is clearly of some importance to produce and test a milk substitute, very similar in composition to rats' milk, to attempt to resolve these problems.

This research was funded from grants to Professor John Dobbing by the Medical Research Council and the National Fund for Research into Crippling Diseases. The authors are extremely grateful to Ted Hall (North Carolina Department of Mental Health) for tutoring J.L.S. in the artificial-rearing technique, to our dissection team, Maureen Leng, Sian Nash, Ann Hutchinson and Angela Tonge, and to Francoise Salomon for determining the dry weights of organs. They also thank the many people with whom they have had helpful discussions during the course of this work, most notably Jaime Diaz (University of Washington, Seattle) and John Edmond and Nancy Sonnenberg (University of California, Los Angeles).

\section{REFERENCES}

Ackerman, S. H. \& Shindledecker, R. (1978). Devl Psychobiol. 11, 385.

Adlard, B. P. F., Dobbing, J. \& Smart, J. L. (1973). Biol. Neonate 23, 95.

Anderson, T. A., Rafferty, C. J., Birkhofer, K. K. \& Fomon, S. J. (1980). J. Nutr. 110, 2374.

Barnett, S. A. \& Burn, J. (1967). Nature, Lond. 213, 150.

Coward, W. A., Cole, T. J., Gerber, H., Roberts, S. B. \& Fleet, I. (1982). Pflügers Arch. 393, 344.

Daly, M. (1973). Br. J. Psychol. 64, 435.

Diaz, J., Moore, E., Petracca, F., Schacher, J. \& Stamper, C. (1981). Physiol. Behav. 27, 1103.

Diaz, J., Samson, H., Kessler, D., Stamper, C., Moore, E., Robisch, E. \& Hodson, A. (1980). Ped. Res. 14, 595.

Diaz, J. \& Schain, R. J. (1978). Science, N.Y. 199, 90.

Dobbing, J. (1981). In Infant and Child Feeding, p. 399 [A. M. Thompson, J. Bond, L. Filer, G. A. Leveille and W. Weil, editors]. London: Academic Press.

Godbole, V. Y., Grundleger, M. L., Pasquine, T. A. \& Thenen, S. W. (1981). J. Nutr. 111, 480.

Hahn, P. (1979). In Nutrition: Pre- and Postnatal Development, p. 1 [M. Winick, editor]. New York: Plenum Press.

Hall, W. G. (1975). Science, N.Y. 190, 1313.

Katz, H. B., Rosett, R. E. \& Ostwald, R. (1979). Devl Psychobiol. 12, 305.

Levine, S. (1959). Can. J. Psychol. 13, 243.

Levine, S. \& Mullins, R. F. (1968). In Early Experience and Behavior, p. 168 [G. Newton and S. Levine, editors]. Springfield, I11.: C. C. Thomas.

Levitsky, D. A. \& Barnes, R. H. (1970). Nature, Lond. 225, 468.

McCance, R. A. (1976). Proc. Nutr. Soc. 35, 309.

Messer, M., Thoman, E. B., Terrasa, A. G. \& Dallman, P. R. (1969). J. Nutr. 98, 404.

Plaut, S. M. (1970). Devl Psychobiol. 3, 157.

Russell, P. A. (1971). Psychol. Bull. 75, 192.

Smart, J. L. (1980). Devl Psychobiol. 13, 431.

Smart, J. L. \& Dobbing, J. (1971). Brain Res. 33, 303.

Smart, J. L., Dobbing, J., Adlard, B. P. F., Lynch, A. \& Sands, J. (1973). J. Nutr. 103, 1327.

Smart, J. L., Katz, H. B. \& Stephens, D. N. (1981), Proc. Nutr. Soc. 40,64A.

Smart, J. L., Stephens, D. N. \& Katz, H. B. (1982). Proc. Nutr. Soc. 41, 12A.

Sonnenberg, N., Bergstrom, J. D., Ha, Y. H. \& Edmond, J. (1982). J. Nutr. 112, 1506.

Whatson, T. S. \& Smart, J. L. (1978). Physiol. Behav. 20,749.

Widdowson, E. M. \& McCance, R. A. (1960). Proc. Roy. Soc. B 152, 188. 